\title{
Retinal nerve fibre layer thickness correlates with brain white matter damage in multiple sclerosis: A combined optical coherence tomography and diffusion tensor imaging study
}

Multiple Sclerosis Journal 2014, Vol. 20(14) 1904-1907 DOI: 10.1177 1352458514535128

(C) The Author(s), 2014. Reprints and permissions: http://www.sagepub.co.uk/ journalsPermissions.nav

\author{
Michael Scheel, Carsten Finke, Timm Oberwahrenbrock, Alina Freing, Luisa-Maria Pech, \\ Jeremias Schlichting, Carina Sömmer, Jens Wuerfel, Friedemann Paul and Alexander U Brandt
}

Abstract: We investigated the association of retinal nerve fibre layer thickness (RNFL) with white matter damage assessed by diffusion tensor imaging (DTI). Forty-four MS patients and 30 healthy subjects underwent optical coherence tomography. DTI was analysed with a voxel-based whole brain and regionbased analysis of optic radiation, corpus callosum and further white matter. Correlations between RNFL, fractional anisotropy (FA) and other DTI-based parameters were assessed in patients and controls. RNFL correlated with optic radiation FA, but also with corpus callosum and remaining white matter FA. Our findings demonstrate that RNFL changes indicate white matter damage exceeding the visual pathway.

Keywords: Multiple sclerosis, optical coherence tomography, diffusion tensor imaging, optic nerve, optic radiation, retina

Date received: 26 January 2014; revised: 16 April 2014; accepted: 20 April 2014

Introduction

Retinal nerve fibre layer (RNFL) thickness from optical coherence tomography (OCT) has been suggested as a biomarker for white matter damage in multiple sclerosis (MS). ${ }^{1}$ Previous studies have reported that RNFL thinning occurs early in MS and also in patients with mild clinical symptoms. ${ }^{2}$ Furthermore, they demonstrated that RNFL thinning occurs independently of optic neuritis (ON). ${ }^{1,2}$

Diffusion tensor imaging (DTI) is an MRI technique based on the orientation-dependent analysis of water diffusion and reveals tissue microstructure. Quantitative parameters, most commonly fractional anisotropy (FA), can be derived from DTI reflecting microstructural integrity of white matter. ${ }^{3}$

Previous studies using OCT and DTI demonstrated an association between RNFL thickness and microstructural integrity in the visual pathway, i.e. the optic nerve, tract and radiation..$^{4-8}$ We investigated in a combined OCT/DTI study whether RNFL thinning may also reflect whole brain white matter damage beyond the visual pathway.
Patients and methods

\section{Subjects}

The local ethics committee approved the study and all participants gave written informed consent. We recruited 44 patients with MS. Patients had to be on stable immunomodulatory therapy for $>3$ months with no acute relapse for at least three months prior to enrolment. Exclusion criteria were pregnancy, metal implants, hearing impairment and severe physical or cognitive impairment. Neurological disability was assessed according to the Expanded Disability Status Scale (EDSS). A group of healthy controls $(n=30)$ received the same measurement protocol (Table 1).

\section{$O C T$}

OCT was performed with a Heidelberg Spectralis SD-OCT (Heidelberg Engineering, Germany) using the peripapillary ring scan, which determines RNFL thickness around the optic nerve head in a 12-degree circle. Image quality was assessed using the OSCAR-IB criteria with all scans passing.
Correspondence to: Alexander U Brandt NeuroCure Clinical Research Center, Charité - Universitätsmedizin Berlin, Charitéplatz 1, 10117 Berlin, Germany. alexander.brandt@charite. de

Michael Scheel Institute of Neuroradiology, Charité - Universitätsmedizin Berlin, Germany

Carsten Finke Charité - Universitätsmedizin Berlin, Germany

Timm Oberwahrenbrock Luisa-Maria Pech Jeremias Schlichting Carina Sömmer Alexander U Brandt NeuroCure Clinical Research Center, Charité - Universitätsmedizin Berlin, Germany

Friedemann Paul Charité - Universitätsmedizin Berlin, Germany/NeuroCure Clinical Research Center,

Charité - Universitätsmedizin Berlin, Germany

Alina Freing

Institute of Interventional and Diagnostic Neuroradiology,

University Medicine Göttingen, Germany

Jens Wuerfel

NeuroCure Clinical Research Center, Charité - Universitätsmedizin Berlin, Germany/Institute of Interventional and Diagnostic Neuroradiology, University

Medicine Göttingen, Germany 
Table 1. Demographics of patient and control group.

\begin{tabular}{|c|c|c|c|c|}
\hline & & MS patients & Controls & Test \\
\hline \multirow[t]{5}{*}{ Subjects } & $N(\mathrm{~F} / \mathrm{M})$ & $44(26 / 18)$ & $30(20 / 10)$ & \\
\hline & RRMS/SPMS & $39 / 5$ & & $p=0.51$ \\
\hline & $N$ without $\mathrm{ON}$ history & 12 & - & Chi-square \\
\hline & $N$ ON history unilateral & 14 & - & \\
\hline & $N$ ON history bilateral & 18 & - & \\
\hline \multirow[t]{2}{*}{ Eyes } & $N$ total & 88 & 60 & \\
\hline & $N$ with history of $\mathrm{ON}$ & 50 & - & \\
\hline $\begin{array}{l}\text { Age } \\
\text { (years) }\end{array}$ & Mean (SD) & $40.0(12.9)$ & $45.9(10.1)$ & $\begin{array}{l}p=0.04 \\
\text { Unpaired } t \text {-test }\end{array}$ \\
\hline EDSS & Median (range) & $2(0-6)$ & & \\
\hline
\end{tabular}

Table 2. GEE correlational analysis results between RNFL and FA.

\begin{tabular}{lllll}
\hline \multirow{2}{*}{ Region } & \multicolumn{4}{l}{ Regression coefficient B (and std. error) from GEE model } \\
\cline { 2 - 5 } & FA & MD & PD & RD \\
\hline Optic radiation (OR) & $1.41(0.35)^{\mathrm{c}}$ & $-0.74(0.45)$ & $0.76(0.52)$ & $-1.48(0.51)^{\mathrm{b}}$ \\
Central corpus callosum (CC) & $1.33(0.38)^{\mathrm{c}}$ & $-1.27(0.70)$ & $-0.14(0.77)$ & $-1.84(0.70)^{\mathrm{b}}$ \\
Whole skeleton (OR and CC excluded) & $0.53(0.17)^{\mathrm{c}}$ & $-0.45(0.26)$ & $-0.01(0.02)$ & $-0.60(0.28)^{\mathrm{a}}$ \\
\hline GEE: generalised estimating equation regression analysis of retinal nerve fibre layer thickness (RNFL) with diffusion tensor imaging \\
(DTI) parameters - fractional anisotropy (FA), mean diffusivity (MD), parallel diffusivity (PD) and radial diffusivity (RD). ${ }^{\mathrm{a}} p<0.05 ;$ \\
b $p<0.01 ;{ }^{c} p<0.001$.
\end{tabular}

\section{DTI}

DTI data were acquired at 3 T (TIM TRIO, Siemens Erlangen, Germany) using a 12-channel head coil. A single-shot echo planar imaging DTI sequence was applied (repetition time (TR)/echo time (TE) = $7500 / 86 \mathrm{~ms}$; field of view $(\mathrm{FOV})=240 \mathrm{~mm}$; matrix $96 \times 96,61$ slices without gap, slice thickness $=2.3$ $\mathrm{mm}, 64$ non-colinear directions, b-value $=1000 \mathrm{~s} /$ $\mathrm{mm}^{2}$, parallel imaging generalised autocalibrating partially parallel acquisitions (GRAPPA) factor 2, acquisition time 8:32 minutes).

\section{Tract-based spatial statistics (TBSS)}

DTI processing was performed with FMRIB Software Library (FSL). ${ }^{9}$ Pre-processing included brain extraction and eddy current distortion correction. Fractional anisotropy (FA), mean diffusivity (MD) and eigenvalue maps were calculated by fitting a linear tensor model to the diffusion data. Parallel diffusivity (PD) was defined as first eigenvalue, and radial diffusivity (RD) was calculated as the mean of the second and third eigenvalue. The resulting maps were further processed in a TBSS analysis. For region of interest
(ROI)-based analysis we overlaid the TBSS skeleton with masks from the Johns Hopkins University white matter atlas (implemented in FSL). We restricted our analysis to masks of the optic radiation (OR), the central corpus callosum and the remainder of the whole skeleton after excluding these two masks. We chose the corpus callosum as it is predominantly affected in MS but functionally separated from the visual system (note that the splenium/forceps major as the interconnection between both occipital cortices was not part of this mask).

\section{Statistical analysis}

Correlations between mean values of DTI parameters and RNFL were analysed with generalised estimating equation models (GEE) to account for intra-patient/ inter-eye effects (R-project 3.0.2 with geepack). Age and $\mathrm{ON}$ history (yes/no) of each eye were included as covariates. For statistical analysis of TBSS results we used FSL's randomise tool, which uses a permutationbased, threshold-free cluster-detection algorithm. We separately tested control and patient groups for correlation between DTI parameters and mean RNFL 
(i.e. the average of both eyes for each subject) including age as a covariate. A multistep linear regression was used to determine $\mathrm{R}^{2}$-changes from each ROI for the prediction of RNFL.

\section{Results}

TBSS analysis showed a significant positive correlation between RNFL and FA and RD along the OR but also beyond that region (Figure 1(a)). The correlation analysis between RNFL and DTI parameters inside the OR, central corpus callosum and whole skeleton (including and excluding these two regions) confirmed the TBSS results in MS patients (Table 2 and Figure 1(a)). We observed a comparably strong association of RNFL and DTI parameters within the OR and central corpus callosum (Figure 1(b), (c)). A weaker association was demonstrated in the rest of the skeleton (Figure $1(\mathrm{~d}))$. In a multistep regression model, age $\left(R^{2}=0.124\right.$, $p<0.001)$, whole skeleton $\left(R^{2}\right.$-change $=0.1035, p=$ $0.001)$, corpus callosum $\left(R^{2}\right.$-change $=0.0334, p=$ $0.055)$, OR $\left(R^{2}\right.$-change $\left.=0.0673, p=0.005\right)$ and history of ON $\left(R^{2}\right.$-change $\left.=0.0228, p=0.094\right)$ contributed to a total $R^{2}=0.351$ of RNFL prediction.

No correlation was found between RNFL and FA in the control group either in the TBSS or in the regionbased analysis.

\section{Discussion}

We investigated the association between RNFL and brain white matter integrity assessed by DTI in MS. Our study showed a significant correlation of RNFL with WM tracts functionally separated from the visual system. Additionally our study confirmed a good correlation between RNFL and FA inside the optic OR. In the region-based analysis we confirmed an association between RNFL and FA values inside the central corpus callosum and also in the remainder of the white matter. In contrast, no such associations were detected in the control group. This suggests that the relation between RNFL and FA found in MS is related to disease pathology, supporting that RNFL may serve as a biomarker for estimation of white matter damage in MS.

The association of RNFL and FA in the OR falls in line with findings from a recent study ${ }^{10}$ that investigated underlying pathophysiological mechanisms, i.e. primary neurodegeneration due to tract disruption and secondary trans-synaptic neurodegeneration. This suggests that trans-synaptic neurodegeneration could explain the link between low RNFL thickness and low FA values in the OR.

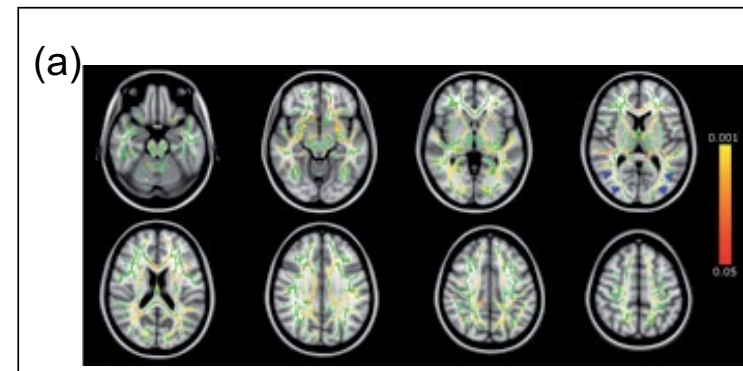

(b)

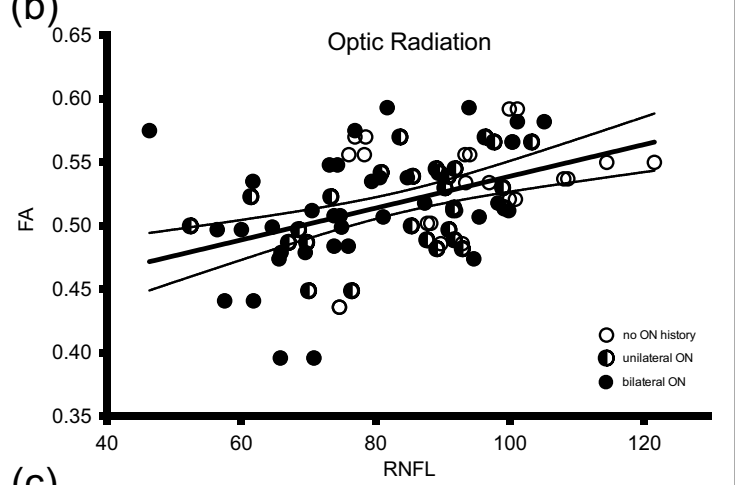

(c)

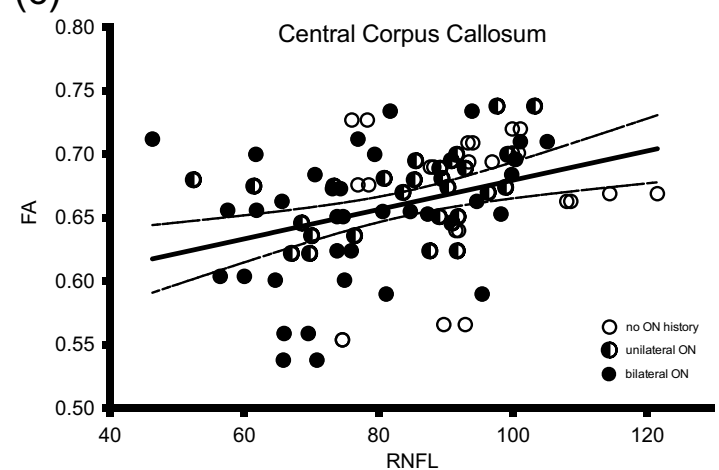

(d)

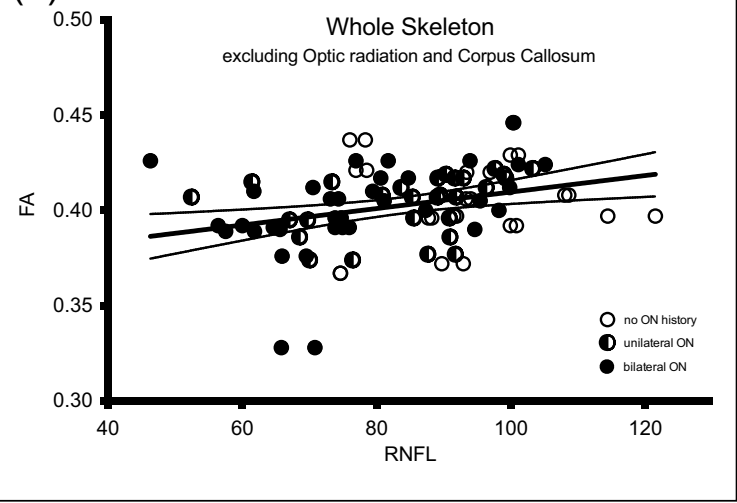

Figure 1. (a) TBSS results for positive correlation between RNFL and FA in MS patients. TBSS skeleton in green; areas with significant correlation in yellow-red; blue arrows indicate the optic radiation. (b)-(d) Scatter plots of RNFL vs. FA in different white matter regions in MS patients including regression line and $95 \%$ confidence interval. Points are coded with regard to optic neuritis history (see legend).

TBSS: tract-based spatial statistics; RNFL: retinal nerve fibre layer thickness; FA: fractional anisotropy; MS: multiple sclerosis. 
One limitation of our study is the limited sample size that prohibited separate analyses of eyes with and without an ON history. Instead, we included ON history as a covariate in our models. Also, we applied a TBSS analysis instead of tractography-based white matter delineation. However, tractography depends on ROI positioning, and lesions along a certain tract may influence tractography results. In our opinion TBSS provides a more objective analysis in the given situation.

\section{Acknowledgements}

We thank Cynthia Kraut and Susan Pikol for excellent technical assistance.

\section{Conflicts of interest}

None declared.

\section{Funding}

This work was supported by the German Research Foundation (DFG grant Exc. 257 to FP). MS is supported by the Friedrich C. Luft Clinical Scientist Pilot Program funded by Volkswagen Foundation and Charité Foundation.

\section{References}

1. Oberwahrenbrock T, Schippling S, Ringelstein M, et al. Retinal damage in multiple sclerosis disease subtypes measured by high-resolution optical coherence tomography. Mult Scler Int 2012; 2012: 530305 .

2. Oberwahrenbrock T, Ringelstein M, Jentschke S, et al. Retinal ganglion cell and inner plexiform layer thinning in clinically isolated syndrome. Mult Scler 2013; 19: 1887-1895.

3. Pierpaoli $\mathrm{C}$ and Basser PJ. Toward a quantitative assessment of diffusion anisotropy. Magn Reson Med 1996; 36: 893-906.

4. Kolbe SC, Marriott M, Walt Av, et al. Diffusion tensor imaging correlates of visual impairment in multiple sclerosis and chronic optic neuritis. Invest Ophthalmol Vis Sci 2012; 53: 825-832.

5. Smith SA, Williams ZR, Ratchford JN, et al. Diffusion tensor imaging of the optic nerve in multiple sclerosis: Association with retinal damage and visual disability. AJNR Am J Neuroradiol 2011; 32: $1662-1668$.

6. Dasenbrock HH, Smith SA, Ozturk A, et al. Diffusion tensor imaging of the optic tracts in multiple sclerosis: Association with retinal thinning and visual disability. J Neuroimaging 2011; 21: e41-e49.

7. Reich DS, Smith SA, Gordon-Lipkin EM, et al. Damage to the optic radiation in multiple sclerosis is associated with retinal injury and visual disability. Arch Neurol 2009; 66: 998-1006.

8. Naismith RT, Xu J, Tutlam NT, et al. Disability in optic neuritis correlates with diffusion tensorderived directional diffusivities. Neurology 2009; 72 : 589-594.

9. Jenkinson M, Beckmann CF, Behrens TE, et al. FSL. Neuroimage 2012; 62: 782-790.

10. Rocca MA, Mesaros S, Preziosa P, et al. Wallerian and trans-synaptic degeneration contribute to optic radiation damage in multiple sclerosis: A diffusion tensor MRI study. Mult Scler 2013; 19: 1610-1617.
Visit SAGE journals online http://msj.sagepub.com

(SAGE journals 